\title{
Analytic Procedures: A Holdback-vetting Forecasting Model
}

\author{
Edward J. Lusk ${ }^{1}$ \\ ${ }^{1}$ The School of Business and Economics, The State University of New York (SUNY) at Plattsburgh, Plattsburgh, NY, \\ USA
}

Correspondence: E. J. Lusk, SBE SUNY Plattsburgh 101 Broad St. Plattsburgh, NY, USA 12901, USA.

Received: December 13, 2016

Accepted: January 5, $2017 \quad$ Available online: January 11, 2017

doi:10.11114/afa.v3i1.2139

URL: http://dx.doi.org/10.11114/afa.v3i1.2139

\begin{abstract}
Introduction: Forecasting is now a best practices requirement for PCAOB audits. This is clear from AS 5 where Analytic Procedures are now a part of the Planning and Substantive Phases of the certification audits. In this regard, we are encouraged by the "On the Go Stores" AP case offered by the AICPA, and have extended their case illustration of AP treatments.

Study Précis: In our presentation, we initially consider the OLS Regression model utilized in the AICPA case and offer a vetting protocol to rationalize the use of this forecasting model in the AP phases. Then we move to a disposition analysis stage where the forecast information is posed in relief to the actual client value so as to ascertain if Extended Procedure investigations would be warranted.
\end{abstract}

Results: We offer three Confidence Intervals drawn from the OLS modeling system that are formed from the Fixed Effect, Random Effects, and finally the Excel Platform for the 95\% CI parameter set.

Impact: The protocol set is programmed in an open-access VBA Decision Support System which is available free as a download with no restriction on its use.

Keywords: OLS regression, disposition, extended procedures

\section{Introduction and Context}

Analytical Procedures [AP] were an integral part of the 2002 roll-out of the re-tooled audit standards labelled as Audit Standards 2 [AS2: Note 1] of the then newly constituted Public Company Accounting Oversight Board [PCAOB] (Sarbanes-Oxley: Pub. L. 107-204, 116 Stat/ 745 (2002). PCAOB is the Public Accounting licensing arm of the Security \& Exchange Commission [SEC]. A recent definition of the AP is offered by The Auditing Standards Board of the American Institute of Certified Public Accountants [AICPA] which follows the definition of AP as offered by the International Auditing and Assurance Standards Board [IAASB: para04;p.436: AU-C:ISA 520 (in conjunction with International Standards on Auditing [ISA] 2009]:

"For the purposes of the ISAs, the term "analytical procedures" means evaluations of financial information through analysis of plausible relationships among both financial and non-financial data. Analytical procedures also encompass such investigation as is necessary of identified fluctuations or relationships that are inconsistent with other relevant information or that differ from expected values by a significant amount."

\subsection{Executing the AP Phase}

The richness of the AP panoply needed in the best practices execution of the certified audit has increased in an explosive manner since 2002. See Imoniana, Antunes, Mattos \& Maciel (2012). This growth is due in large part to enhancements in technical data processing functionalities such as: Commercial Software, Institutional Shareware, Decision Support Systems [DSS], and innovative uses of the myriad Statistical Techniques called out by the Big-Data world of PCAOB audit engagements. See Akkaya \& Uzar (2011).

In 2007, AS5, the update of AS2, formally introduced the best practices requirement that AP needs to be executed at the Planning Stage [Phase 1] and also at the Substantive Phase [Phase 3]. Usually, the disposition of the difference between the Phase 1 and Phase 2 information sets generates an impact in the Report Writing Phase [Phase 4]. This AP requirement directly and simply rationalizes the use of Forecasts at the Planning Phase and the examination of variances from Forecasted Expectations relative to actual reported values in the audit client's Financial Statements. This is just the 
post-audit phase which is well documented in the forecasting literature. See Kennedy \& Mills (1992); O’Donnell \& Perkins (2011); also Stanley, Brandon \& McMillan. (2015).

We call this the Disposition Analysis where:

directional variances of: [AP-Forecasted Account values from YE Actual Account Values] are considered by the auditor in forming an opinion as to the need for further investigation, usually called Extended Procedure Investigations in certifying the Financials of the audit client.

The information flows that define the AP Disposition Analysis are expressed in Figure A.

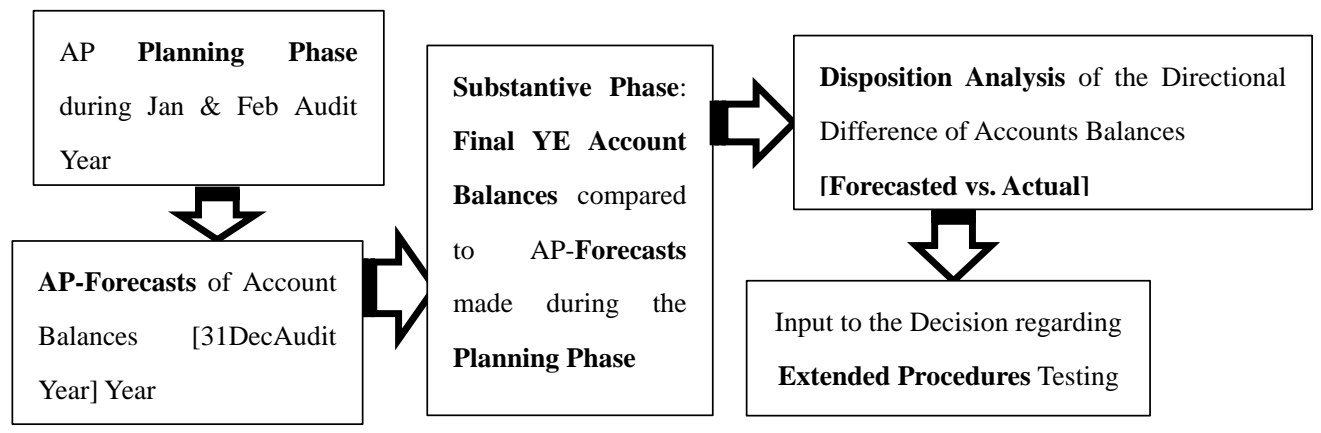

Figure A. AP Disposition Analysis Employing a Forecasting Protocol

\subsection{The Committee of Sponsoring Organisations of the Treadway Commission [COSO] Context}

The above definition of AP and the related Disposition Analysis [DA] Phase clearly are focused on the magnitude of possible errors and their related materiality. However, as a logical extension, AP and DA also can impact the Interim Testing Phase of the Audit [Phase 2] where the COSO testing required by AS5: Section 404 is serviced. Specifically, consider a direct quote of: PCAOB: AS 5 (2007) in Appendix B Special Topics, p. A1-45, Sub-Section B4: Tests of Controls in an Audit of Financial Statements where the PCAOB offers the following:

"To express an opinion on the financial statements, the auditor ordinarily performs tests of controls and substantive procedures. The objective of the tests of controls the auditor performs for this purpose is to assess control risk. To assess control risk for specific financial statement assertions at less than the maximum, the auditor is required to obtain evidence that the relevant controls operated effectively during the entire period upon which the auditor plans to place reliance on those controls."

The emphasis of this citation is clearly on the magnitude and materiality but also on the control affected by management over financial reporting. In this COSO domain, the auditor is focused on the nature and processing integrity of the Accounting Information System [AIS] and the related segregation of duties which, of course, has an important impact on the assessed risk level of the audit. In this Phase 2, COSO context DA may be used to check the nature of the AIS errors that formed the difference between the Forecasted \& the Actual values during the Interim phase of the audit. "Grosso Modo" then Disposition Analysis is also relevant in all the phases of the audit.

These dual foci offered by the PCAOB require that two opinions are expressed in the client's certified audit report. The Financial Report speaks to the fairness of the financial statements regarding the Magnitude \& Materiality of possible errors and the COSO Report addresses the Adequacy of Management System of Control over Financial Reporting. As suggested above, both of these reporting imperatives can be serviced using a forecasting model and the related disposition protocol.

To form this DA, we will focus on the AP: Planning \& Substantive Phases as they were used in the AICPA initiative - the Clarity Project.[Note 2] Specifically, we will model our presentation to follow the AP "work-up" as offered in the "On the Go Stores" case as reported in AICPA (2012): Analytical Procedures.

DA is the pre-curser of making the decision regarding the further analysis of the accounts due to the results of the DA or what is usually called an Extended Procedures [EP] investigation. EP is complicated and relates to the inherent and control residual risk and, of course, the reliability of the forecasting method used in the DA Phase. For an excellent discussion of EP from a practical perspective see: Bettauer (1975). This is the point of departure of our research.

We are encouraged by and appreciate the AICPA's "On the Go Stores" case and will use their AP tutorial as the model for elaborating the DA as previewed in Figure 1. In this regard, we will also employ the OLS two-parameter linear Regression model [RM] used in the "On the Go Stores" case. 


\section{Précis of the Forecasting AP-Disposition Protocol [FAPDP] Study}

Extending the "On the Go Stores" example we will:

1. Provide an Overview of the logic of the Disposition Analysis [DA] in the audit context.

2. Introduce and recommend various pre-analysis data preparation steps that experience recommends are often needed in the usual data download context where longitudinal information of the clients' selective sensitive accounts is used in the AP planning phase.

3. Offer a Holdback Validation Protocol that will be suggested as conditioning information and so offering validation insights regarding using the RM in the AP-loop. This will be the first phase of the FAPDP.

4. Present, in detail, three statistical confidence intervals that are part of the RM context. In this second phase we will use these three confidence intervals as triage screens to offer summary information regarding launching an AP Extended Procedures examination. All of the information that will be presented is available in a DSS called FAPDP: DSS that is available free as a download without restriction on its use from the author.

5. Provide a detailed illustration of the FAPDP, following the presentation logistic of the "On the Go Stores" offered by the AICPA.

\section{The Components and Functionalities of the FAPDP: The Overview}

In this section, we offer an overview of the FAPDP. In the following section, we will present the notation that may aid in the VBA-code development of the FAPDP should modifications of the FAPDP-DSS be necessary.

\subsection{Audit Context \& Account Selection}

The fundamental focus of the planning requirement of the AP phases is to aid the auditor in the making the critical decision regarding the use of EP. Underlying the PCAOB's AP imperative is the intent to create screening information, informed by audit experience and common sense, so as to condition the EP decision in such a way that EP are effective and efficient. In the effective domain we note that there are often thousands of accounts each of which has tens of thousands of data items; this is the norm in the Big Data world of PCAOB audits. As Christensen, Elder \& Glover (2015) suggest, the auditor must have an "objective" platform for selecting accounts to create audit evidence. In this regard it is usually the case that the auditor first selects a subset of Sensitive Accounts from the client's active set of accounts; Sensitive Accounts are those that could affect the Current Ratio or Cash Flow from Operations. For the efficiency context the question is: Which of this sub-set of Sensitive Accounts are justifiable candidates to investigate using EP at the Substantive and/or the COSO phases? A logical selection model is to use the DA protocol-to wit: develop a reliable Expectation using the RM and then collect actual client YE-values. At this juncture, the auditor will select a set of accounts for EP investigation where there are important directional differences in the DA phase. This is what we have argued above where the Extended Procedures is conditioned on the Disposition Analysis or EP[DA].

\subsection{Data Preparation}

The next issue that usually needs to be addressed is Missing Data and Anomalous data points in the downloaded time series stream. Surprisingly, downloads of data that would satisfy the time series modeling requirements often have missing data points and/or values that are not likely to fit the usual projection profiling requirements; one usually terms these anomalous data points as outliers. These are issues as the time stream must be complete; so missing data are not acceptable and outliers will perturb model projections. This is particularly true when the projection model is the RM model that we will be using following on the AICPA AP case. There are a few techniques that are used in forecasting that deal with: Missing Data and Anomalous data points in the Data Stream.

Such data issues are usually dealt with by: Blending, Median or Regression Filling. Blending replaces the missing value by the average of the adjacent or neighboring points. Such a replacement protocol addresses the "short"-run trajectory of the data stream and is often used where there seems to be temporal idiosyncrasies or non-systemic-event periodic changes in the data stream-this is sometimes referred to as short-term smoothing. Note, if there are definitive seasonality or regular cycles in the data, then the RM model is not recommended. In this case, the Winters' class of models which are seasonal versions of the Holt/AIRMA $(0,2,2)$ models are recommended. See Box \& Jenkins (1976). Treating these models is a separate topic not addressed in this paper. Median filling installs the Median of the Data as the missing values. This is sometimes used when Winsorizing is not used as the Median is not highly sensitive to non-symmetric imbalances in the histo-profile of the data. See Armstrong \& Collopy (1992). The Median fill seems to work best for ergodic time series. However, if there is a trend in the downloaded data stream, then, as the Median is relatively insensitive to the trend, it usually results in a perturbation of the slope parameters of the OLS model and so is not recommended. The Regression fill installs as the data point the time-oriented regression point. Where there is likely to be a long-run trend the regression fill is preferred. We have programmed these three filling options in the FAPDP-DSS. 


\subsection{Holdback Validation of the OLS Model}

It should not be assumed that a particular forecasting model can be relied upon to create relevant audit information in the DA phase. This is clear as directional differences can be caused by poor forecasts created by inappropriate models. This is an issue that was not considered in the otherwise excellent "On the Go Stores" case. For this reason we will address validation or vetting of the choice of the forecasting model used in the FAPDP. According to extensive research over the last four decades a simple, effective, efficient and extensively used way of benchmarking forecasting activity is the holdback validation of the forecasting model. See Makridakis et al. (1982), continuing with the work of Collopy \& Armstrong (1992), and recently with the complexity calibration work of Adya \& Lusk (2016).

In this regard, we offer, as the validation protocol for the OLS, the following holdback protocol. Recall, to begin the process the auditor has downloaded a sensitive account from the client. It will then be modified for Missing Date and/or Anomalous data as needed. From this "fixed" data set, the auditor will hold back the last two data points in the longitudinal dataset. This stage also has disposition aspects in that we can compare the held back points to the forecast values of the RM model. To enrich the analysis in a robustness sense, we will take advantage of the fact that the RM model can be used to develop various $(1-\alpha)$ Confidence Intervals $[\mathrm{CI}]$ for the forecasted value. These $\mathrm{CI}$ will be used both in the vetting of the RM and also in the DA. The validation or vetting of the RM will be founded if the two holdback points are both in their selected CI generated using the reduced time series. If the holdback points are both in their CI, then the auditor will be reasonably justified in assuming that the RM model is not inappropriate for the AP testing. If both holdback points are NOT in their selected CI, then the likely case is that the RM model is not appropriate for generating reliable audit evidence relative to the decision regarding extended procedures testing. If one point is in and one point is not in the respective (1- $\alpha)$ Confidence Intervals, then the auditor should collect related confirmatory information to test the appropriateness of the RM.

\subsection{Confidence Interval Screening: the Disposition Analysis}

The final aspect of this FAPDP model is, of course, the reason that we have developed all the above steps. This then is the final phase where the Disposition Analysis will be completed and also the Extended Procedure information will be developed. If the auditor is at this stage, then the RM has been accepted as the forecasting model. In this case, ALL of the data will be used and so the two hold back data points will be "added back" into the data stream. This full data stream will be used to forecast the next data account point. In this regard, for purposes of the DA and also the EP, we will be using the selected (1- $\alpha$ )Confidence Interval to make the judgment regarding the Disposition of the YE account value and the forecasted value as suggested in the DA phase.

Previously we mentioned that there are in fact various (1- $\alpha$ )Confidence Intervals that may be employed in the vetting or the DA[EP] phases. This will greatly enhance the relevance of the information generated at the DA and the EP phases as this can be viewed as a robustness test of the FAPDP. We will elaborate and develop the required information on these three $(1-\alpha)$ Confidence Interval possibilities in the next section. It is sufficient to note here that the actual client data value at the COSO or Substantive stages is evaluated by where it is located in three confidence intervals.

Following the above section, where we have given the overview context of the FAPDP model, we will now present the notation that will be useful in expressing the details of the model and so may be used in VBA-coding or modification of the DSS-FAPDP.

\section{The FAPDP components and Functionalities: The Details}

In this section, there are various topics treated. They are presented in a logical logistical order and then illustrated by a case.

\subsection{Audit Context \& Account Selection}

The auditor has the population of client accounts-i.e., the available "population" General Ledger account entry set from the AIS. In most template software systems this is called the active Chart of Accounts [CA: $\left.\left\{A_{i}\right\} i=1,---, N\right]$. From the CA, the auditor identifies or selects the set of Sensitive Accounts $\left[\mathrm{CA}:\left\{S A_{j}\right\} j=1,---, n\right]$ where: typically, effectively by logic, $\mathrm{N} \gg>\mathrm{n}$. Next, the auditor will select a few SA to use in the FAPDP protocol. Note this subset as: $\mathrm{SA}:\left\{S A_{h t}^{\text {test }}\right\}\left[h=1,---, n_{\text {test }} ; t=1,---, k_{h}\right]$ where: $\mathrm{t}$ is the time series index. This is to say $t \subset$ [Ordered:\{I\}], the end point of which, $k_{h}$, may be variable depending on the particular Sensitive Account $[h]$ selected. Usually, it is the case that: $N>>>n \gg n_{\text {test }}$.

\subsection{Data Preparation}

For each $S A_{h t}^{\text {test }}$, the auditor will identify any missing time points and also any anomalous/outlier points in the client download. They will be "corrected/prepared" using the appropriate Blend, Median, or Regression fill-protocols 
discussed above. Call these corrected series, for simplicity, $Y_{h t}$. Next the auditor chooses a time series accrual time period: $Y_{h t} t=1,---, k_{h}$; where $k_{h} \subset$ in the crisp range [13 to $\left.Y_{h t}[E H]\right]$ i.e., at least 13 points from the time of the last observation, $k_{h}$, indexing back to the Event Horizon [EH] for $Y_{h t}$. For $Y_{h t} t=1,---, k_{h}$ the holdback RM-validation test will use the accrual $Y_{h t} t=1,---, \Omega_{h},\left(\Omega_{h}+\mathbf{1}\right),\left(\Omega_{h}+2\right)_{=k_{h}}$. Where: the bolded points are the two holdback values from the end of the accrual period. We used 13 points as the lower limit of the data stream referencing the work of Adya \& Lusk (2016) who find that inference for forecasting models, the sort of which we are considering in this paper, is compromised if the data stream has less than 13 time-series points. Therefore, in the holdback stage there should be at least 15 data points accrued as two points are to be held back.

\subsection{Holdback Validation of the RM}

The vetting test is to form the $(1-\alpha)$ Confidence Interval hereafter noted as: $[(1-\alpha)[C I]]$ individually for each of the holdback points. To be clear, the first $[(1-\alpha)[C I]]$ will be formed from the time-series set of $\left\{\Omega_{h}\right\}$ points and used to test $Y_{h\left[t=\left(\Omega_{h}+1\right)\right]}$. The second [(1- $\left.\left.\alpha\right)[C I]\right]$ will be formed from the time series set of $\left\{\Omega_{h}+1\right\}$ points and used to test $Y_{h\left[t=\left(\Omega_{h}+2\right)\right]}$. This holdback phase will provide vetting-information regarding the appropriateness of the RM. As presented in the overview, the logic of this proposed vetting is:

1. If both of the Holdback points are NOT in their respective $(1-\alpha)$ CIs, then this is reasonably clear evidence that the RM may NOT be the best choice in executing the FADPD. For example, it may be that short-run irregularities produce data that are exterior to the linear projection $(1-\alpha)$ CI. This often happens and effectively was the reason for developing "damped-slope" regression models which are of the ARIMA class $(1,1,2)$ See (JMP $^{\circledR}$ (2005:p.615); also such temporal instabilities are the reason that Collopy and Armstrong (1992) utilized a Slope dampening rule set [Rules 89 to 95 in Appendix B] in their seminal RBF paper.

2. If both of the Holdback points are IN their respective $(1-\alpha)$ CIs, then this is reasonably clear evidence that the RM cannot be argued as inappropriate in executing the FADPD.

3. However, if only one point of the set: $\left\{Y_{h\left[t=\left(\Omega_{h}+1\right)\right]} \& Y_{h\left[t=\left(\Omega_{h}+2\right)\right]}\right\}$ is IN the related (1- $\left.\alpha\right)$ CI for the forecast projections, then this may call into question the appropriateness of the RM. In this case, the auditor may continue with due caution and professional skepticism and would seek further confirmatory indications in the DA \& EP phases. A caveat: Our testing experience with the FAPDP is that unless both points are in the $(1-\alpha)$ CI, the OLS model should be rejected as the forecasting model. This is more in the "anecdotal" domain than vetted or confirmed information.

At this point, we need to discuss the usual confidence interval choices for the RM. After we detail these three modeling choices we will suggest how they should be used in the holdback-vetting and then the DA \& EP phases.

\subsubsection{The Forecasting Confidence Interval Choices}

There are three possible CI choices: the Excel ${ }^{\mathrm{TM}}$ "Extreme Parameter" Range profile, the Random Effects profile, or the Fixed Effects profile. To simplify the notation we will drop the $h$ index which identifies a particular time series.

Excel Parameter Range Model The Excel Regression functionality of the Data Analysis Tab allows the creation of the "covering" confidence interval projections individually for the two holdback points as follows:

Extreme Left Side[LowerLimit[LL]] Boundary for the first holdback : $Y_{\left[t=\left(\Omega_{h}+1\right)\right]}$

$$
\hat{Y}_{\text {LowerLimit: }\left(t=\left(\Omega_{h}+1\right)\right)}=\left[\hat{\alpha}_{\Omega}-\left[t_{\frac{\alpha}{2}} \times s_{\epsilon(\alpha)}\right]\right]+\left[\hat{\beta}_{\Omega}-\left[t_{\frac{\alpha}{2}} \times s_{\epsilon(\beta)}\right]\right] \times[\Omega+1]
$$

Extreme Right Side[UpperLimit[UL]] Boundary:

$$
\widehat{Y}_{\operatorname{UpperLimit}\left(t=\left(\Omega_{h}+1\right)\right)}=\left[\hat{\alpha}_{\Omega}+\left[\operatorname{to}_{\frac{\alpha}{2}} \times s_{\epsilon(\alpha)}\right]\right]+\left[\hat{\beta}_{\Omega}+\left[\operatorname{to}_{\frac{\alpha}{2}} \times s_{\epsilon(\beta)}\right]\right] \times[\Omega+1]
$$

Where: $t_{\frac{\alpha}{2}}$ is the t-statistic for inference that has $d f=[(\Omega)-2]$. Recall that the $(\Omega)$ indicates that the regression is performed on the time series less the two holdback points which will then indicate the number of points in the OLS time series fit.

In like fashion, one may form the $(1-\alpha)$ CI for the second or last data value held back : $Y_{h\left[t=\left(\Omega_{h}+2\right)\right]}$ with the usual adjustment in the $d f$.

The Random Effects Model The OLS assumption in this case is that there is random sampling possibility from a 
well-defined population of variable data streams of realizations i.e., the set of:

$$
\left\{Y_{t}^{l} l=\text { Firm Index where } l \text { is large }\right\}
$$

which constitutes a blocked or stratified group of "like" firms. In this case, the auditor believes that the sample estimate is used to produce the forecast of the Expectation of all of the firms in: $\left\{Y_{t}^{l}\right\}$ from this sub-population or the $\mathrm{E}\left[Y_{t}^{l}\right]$. In this case, and given the usual assumptions rationalizing the RM of the time series, where: $t$ is the independent variable time-index, the confidence interval for the projection for the accrual of $\Omega$ data points is:

$$
\hat{Y}_{(t=\Omega+1)} \pm t_{\frac{\alpha}{2}} \times s_{\epsilon} \times \sqrt{\frac{1}{\Omega}+\frac{\left[[(\Omega+1)-\bar{\Omega}]^{2}\right]}{s_{t t}}}
$$

Where: $S_{t t}=\left[\sum t^{2}-\left[\left[\sum t\right]^{2} / \Omega\right]\right]$; where: the degrees of freedom for $t_{\frac{\alpha}{2}}$ is $(\Omega-2), s_{\epsilon}=\operatorname{OLS}[\Omega]: M S E^{1 / 2}$, and $\bar{\Omega}$ is the Mean of the time index for the $\Omega$ data points.

This is a mean estimate over "all" of the elements in the sub-population; this Confidence Interval gives relatively the narrowest precision even though the extrapolation penalty can warp the $\mathrm{CI}$ as the projection drifts away from the middle of the time index.

The Fixed Effect Projection The assumption is that the object of interest is the $\mathrm{j}^{\text {th }}$ firm with a unique set of data stream of realizations: i.e., the set of:

$$
\left\{Y_{t}^{j} j=\text { a particular firm Index }\right\}
$$

Where: this $\mathrm{j}^{\text {th }}$ Firm has longitudinal dummy-variable integrity in comparison to all of the other firms in the population and so the projection is not the average of all the firms but only for that $\mathrm{j}^{\text {th }}$ firm. In this case, and given the usual assumptions rationalizing the OLS Regression of the time series the confidence interval is:

$$
\hat{Y}_{(t=\Omega+1)} \pm t_{\frac{\alpha}{2}} \times s_{\epsilon} \times \sqrt{1+\frac{1}{\Omega}+\frac{\left[[(\Omega+1)-\bar{\Omega}]^{2}\right]}{s_{t t}}}
$$

This may seem a "trivial" modification but increases the width of the confidence interval compared to that of EQ[3] as a multiple ranging almost to a factor!

The next critical issue is to profile the precision of these three CIs so as to make a logical choice of how these CIs should be used in the RM Vetting and the DA \& EP phases. In this regard, it is obvious that the precision of the Random Effects [RE] CI:

$$
\hat{Y}_{(t=\Omega+1)} \pm t_{\frac{\alpha}{2}} \times s_{\epsilon} \times \sqrt{\frac{1}{\Omega}+\frac{\left[[(\Omega+1)-\bar{\Omega}]^{2}\right]}{S_{t t}}}
$$

is less than the precision of the Fixed Effects [FE] CI:

$$
\hat{Y}_{(t=\Omega+1)} \pm t_{\frac{\alpha}{2}} \times s_{\epsilon} \times \sqrt{1+\frac{1}{\Omega}+\frac{\left[[(\Omega+1)-\bar{\Omega}]^{2}\right]}{S_{t t}}}
$$

as the latter has a unit value, i.e., 1.0, and all the values in the root function are positive.

As for the Excel precision: [EQ[2] - EQ[1]]/2 it is not clear for data series often found in the PCAOB account milieu what is the relationship in precision terms for the Excel precision compared to that of EQ[3] \& EQ[4]. One supposes, as EQ[3] is a mean projection, that the precision for this Random Effect CI will be less than for the Excel CI precision as $\mathrm{EQ}[1]$ and EQ[2] are driven by the standard errors of the parameters and all of the projections are only for one period. In this case, the extrapolation "penalty" really does not seem to be a major factor for reasonably long time series. However, no reasonable generalization is possible for the Excel precision compared to that of the Fixed Effects: CI.

4.3.2 Precision Profiles of the Three Confidence Interval To collect original information of these precision relationships, we randomly selected 50-time series used in the Adya \& Lusk (2016) study; these time series datasets have been used in various studies over the last four decades. The crisp range profile of the number of points was: [13:50]. We found for all, $100 \%$, of these 50 series that the precision of the Excel CI was greater than of EQ[3]. Further, the precision of Excel CI was greater than the precision of EQ[4] 78\% of the time.

\subsection{Confidence Interval Screening: The Holdback Vetting and Disposition Analyses}

For this reason, we decided to use the Excel system EQ[1] \& EQ[2] as the discrimination check for the OLS forecasting validation. The logic of this choice is: As the Excel precision is usually the largest this will be the most conservative test 
and so have relatively the lowest False Positive Signal rate that the RM is not appropriate. To be clear, as we are using the widest average precision, if the holdbacks are indeed outside this widest screening CI there is likely to be convincing alert information that the OLS model may not be applicable for the audit context. Therefore, the RM will be vetted under the condition that the holdbacks are not outside the Excel CIs. This being the case then we will move on to the DA phase of the testing

As for the DA and EP phases, we have formed the following triage where the three confidence intervals are presented with the final values produced using all the data points downloaded from the client-e.g., $\left\{Y_{h t} t=1,---, \Omega_{h},\left(\Omega_{h}+1\right),\left(\Omega_{h}+2\right)\right\}$. As this is the DA and the EP phase, the FAPDP: DSS asks the auditor to enter the final client value. In the case to be presented subsequently, and as illustrated in Figure B the client value [CV] was $\$ 1477.00$ for the account under audit which is now expected for the $20^{\text {th }}$ time period.

\begin{tabular}{|c|c|c|c|}
\hline ExcelLLimit & & \multirow[b]{2}{*}{$\$ 1177.89$} & $\$ 1126.02$ \\
\hline FixELLimit & & & \\
\hline RandomELLimit & $\$ 1335.84$ & & \\
\hline & $\$ 1477.00$ & & \\
\hline RandomEULimit & $\$ 1575.07$ & & \\
\hline FixEULimit & & $\$ 1733.02$ & \\
\hline ExcelULimit & & & $\$ 1784.89$ \\
\hline
\end{tabular}

Figure B. The Three 95\% Confidence Intervals for Evaluation of the Client YE Value

The values noted in Figure B are those for the DA \& EP phase where all the data points were used. We will again reference Figure B in the summary section of this paper.

In addition to this graphical presentation of Figure B, the FAPDP:DSS provides, for each of the three practical cases which in this configuration are exclusive, the following Audit Alert information:

1. If the CV is outside the most extreme values of the $95 \% \mathrm{CI}$ triage, then the audit alert produced by the DSS is: In this case, the Actual Client Account value is lower/higher than the lowest/highest AP-Forecasted CI value. This Strongly suggests that Extended Procedures may be warranted.

This not being the case, the next test is:

2. If the $\mathrm{CV}$ is not interior to the smallest $95 \% \mathrm{CI}$, then the audit alert produced by the DSS is: In this case the Actual Client Account value is NOT In the Smallest Interval Confidence Interval Range of AP-Forecasted values. This usually suggests that Extended Procedures may need to be considered.

Finally, the last case is:

3. If the CV is interior to the smallest $95 \% \mathrm{CI}$, in this case: the RE Model, then the audit alert produced by the DSS is: In this case, the Actual Client Account value is In Smallest Confidence Interval Range of AP-Forecasted values. This usually suggests that Extended Procedures may NOT be warranted.

\section{Illustration of the First phase of the FL: APP: Validating the OLS-Model}

All of the information to be presented was produced with the FAPDP: DSS which is available from the author as a download at no cost and with no restrictions. Also, the presentation format for this illustrative example follows the "On the Go Stores" offered by the AICPA (2012). Assume that the In-Charge is interested in the value of the Accounts Receivable for the client at YE 2016. For the forecasting AP model, the OLS model is selected and the time series was downloaded from the client's AIS. The downloaded values are:

Table A. Accounts Receivable Quarterly (000s) to 30Sept2016, n=19

\begin{tabular}{ccccccc}
\hline 331 & 376 & 494 & 630 & 645 & 639 & 634 \\
Missing & 686 & 661 & 750 & 829 & 89100 & 944 \\
1210 & 1271 & 1327 & $\mathbf{1 4 6 6}$ & $\mathbf{1 5 8 5}$ & & \\
\hline
\end{tabular}

\subsection{Fixing the Data Stream}

In this case, there are two issues with the download: the $8^{\text {th }}$ point was missing and the $13^{\text {th }}$ point seems to be anomalous. Using the FAPDP: DSS, the In-Charge decides to blend the Missing point and also to replace the anomalous point as a Blend. This is logical as the time series seems to be drifting upward in a trended manner. In this case, the missing value will be replaced by 660 [Average (634:686)] and 89100 is replaced by 886.50 [Average(829:944)]. This is the "fixed" time series and will now be used in the holdback test. The two holdback points are bolded in Table A. 


\subsection{Vetting the OLS-Forecasting Model}

Using the first 17 points, $\mathrm{n}-2$, and the Excel Regression Platform for the fixed download the OLS regression 95\% CIs for the intercept: $\hat{\alpha}_{0.025}=[170.5830 \& 390.9464]$ and for the slope: $\hat{\beta}_{0.025} \quad$ [42.8454 \& 64.3507]. The validation will be to determine if $\$ 1466$ is in this Excel 95\% covering interval. In this case, the relevant CI for Point $\$ 1466$ is: [[170.583+42.8454×18] \& [390.9464+64.3507×18]] or

The $95 \% \mathrm{CI}$ is [ $\$ 941.80 \& \$ 1549.26$ ] and the first holdback of $\$ 1466$ is in this interval.

Now considering the second holdback point: $\$ 1585$ we find using the first 18 points, $n-1$, from the fixed download the OLS regression 95\% CIs for the intercept: $\hat{\alpha}_{0.025}=[141.3547 \& 371.1813]$ and for the slope: $\hat{\beta}_{0.025}[46.8498 \&$ 68.0821]. In this case, the validation will be to determine if $\$ 1585$ is in this Excel $95 \%$ covering interval. In this case the

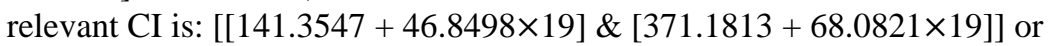

The $95 \%$ CI is [ $\$ 1031.50 \& \$ 1664.74]$ and the second holdback of $\$ 1585$ is in this interval.

As both of the holdbacks are in the validation intervals, this rationalizes that the OLS model seems appropriate for the Extended Procedures test. This being the case, the In-Charge will move to the Disposition Analysis \& Extended Procedures stages.

\subsection{Analytic Procedure Phase}

In these final DA \& EP stages, the auditor will determine the final client account value and then use the 95\% CI triage platform to develop the information for making the EP decision. Assume that the client account value is $C V=\$ 1477$. The auditor enters the CV into the FAPDP: DSS and the full dataset, $k_{h}=19$, as vetted above. The graphic in Figure B is displayed along with the EP-message: In this case, the Actual Client Account value is In Smallest Confidence Interval Range of AP-Forecasted values. This usually suggests that Extended Procedures may NOT be warranted.

Using the full fixed dataset, $k_{h}=19$, the vetted OLS model, and the three CIs from: Excel Model: EQ [1] \& EQ [2], the Fixed Effects Model: EQ[4] and the Random Effects Model EQ[3] the In-Charge sees that the client YE value, \$1477 is interior to the smallest $\mathrm{CI}$ and so this usually suggests that Extended Procedures are not likely to be warranted. As an important performance statistic the Human Information Processing time to produce these results using FAPDP: DSS was less than 1 minute! We are mentioning this as the time to arrive at a fully developed information set in the audit context is critical. Using DSSs is the only practical way to have the possibility to produce a best practices audit in the Big Data world.

\subsection{The Impact of the FAPDP in the Planning and COSO Phases}

Recall that we have taken up the Disposition Analysis [DA] as a natural feature of the Forecasting Model protocol as employed in the AP milieu. We now will offer the linkages for the FAPDP as it could reasonably be used in support of the Financial Statement Opinion and also the COSO Opinion. The connection is immediate as the DA can be used at the Substantive Phase from projections made at the Planning Stage to the Year-End closed client balances. This is the case that the "On the Go Stores" takes up. However, data series at the Planning Phase in January or February of the audit year can equally be used to make a projection to any of the Quarters of the organization's activity or for that matter even on a monthly basis if that were to be the projection set of information. Specifically, if the auditor wants to use the DA for COSO testing at the Interim Phase and that was planned for the June, then the DA would use Quarterly reported information from the 10-Q filings and so project to the Second Quarter or the July filing for the DA testing.

\section{Summary and Outlook}

\subsection{Summary}

In our experience we see that forecasting protocols are very often not part of the creation of audit evidence. If our experience is indicative, then the FAPDP may indeed be a valuable technique in the employ of the In-Charge. We also have had great success in our Audit and Assurance capstone course at the undergraduate level in an AASCB accounting program with this forecasting protocol. Indeed, many of our students take their laptops to professional engagement interviews to "demo" the FAPDP: DSS so as to illustrate their technical empowerment.

The FAPDP is a simple modeling protocol if one uses the DSS that we have developed. Also, the FAPDP fits well with the logic of the PCAOB best practices requirement that AP be used in the creation of audit evidence. Effectively and simply, the FAPDP is just the use of a forecast to make an assessment of the eventual veracity of the client's activity. Additionally, we have included as part of the FAPDP: DSS an open access VBA-Module for Blending and/or Filling where it is needed. Experience in the Big-Data audit-world is that one needs to be skeptical to the point of suspicion about the integrity of downloads.

\subsection{Outlook}

We encourage the PCAOB to follow the lead of the AICPA and produce and publish more Case Analyses where the 
forecasting protocols find productive integration in the creation of audit evidence. Also more academic textbooks need to form the creation of audit evidence around forecasting protocols. Finally, as we have discussed, not all OLS two-parameter models are appropriate for the FAPDP. In fact, we have built into the DSS a reasonability check using a holdback screening protocol as demonstrated above. This begs the creation of other studies that examine the standard OLS model in compression with the ARIMA $(0,2,2)$ /Holt model, the Winter's "Holt" season adaptation, and Judgmental models of the RBF class of Collopy \& Armstrong (1992). Additionally, there are many other GUI-enhancements such as feedback on the nature of the time series, particular transformations in the Box-Cox class, or Post-Audit tracking that can be used productively. See Fildes, Goodwin \& Lawrence (2006); Adya \& Lusk (2013) \& Stanley, Brandon \& McMillan (2015). Finally, recently Adya \& Lusk (2016) have introduced a scoring model for categorizing a time series as to its Complexity. They show clearly that the forecasting performance for Complex time series are dramatically compromised relative to that of Simple series. This has obvious implications for the audit in the employing the FAPDP.

\section{Acknowledgments}

Thanks and appreciation are due to: Dr. H. Wright, Boston University: Department of Mathematics and Statistics, the participants at the SBE Research Workshop at SUNY: Plattsburgh, Mr. Manuel Bern, Deloitte Touche LLP, Audit \& Assurance Services, Frankfurt [Main], Germany and to the anonymous reviewers of the Applied Finance and Accounting for their most helpful suggestions.

\section{References}

Adya, M., \& Lusk, E. (2016). Time series complexity: The development and validation of a Rule-Based complexity scoring technique. Decision Support Systems, on-line. https://doi.org/10.1016/j.dss.2015.12.009

Adya, M., \& Lusk, E. J. (2013) Designing effective forecasting decision support systems: Aligning task complexity and technology support: In Chiang, J. (Ed) Decision Support Systems in Tech. Open Access ISBN: 978-953-51-0799-6.

Akkaya, G., \& Uzar, C. (2011). Data mining: Concept, techniques and applications. Global Science and Technology Forum, 1, 47-50.

American Institute of Certified Public Accountants [AICPA]. (2012). Audit Guide: Analytical Procedures, American Institute of Certified Public Accountants, Inc. New York, NY USA:

https://competency.aicpa.org/media_resources/208125-analytical-procedures-aicpa-audit-guide

Armstrong, J., \& Collopy F. (1992). The selection of error measures for generalizing about forecasting methods: Empirical comparisons. International Journal of Forecasting, 8, 69-80. https://doi.org/10.1016/0169-2070(92)90008-W

Bettauer, A. (1975). Extending audit procedures: When and how. Journal of Accountancy, 11, 69-72.

Box, G. E. P., \& Jenkins, G. M. (1976). Time Series Analysis, Forecasting and Control. Holden-Day, San Francisco, $3^{\text {rd }} \mathrm{E}$.

Christensen, B., Elder, R., \& Glover, S. (2015). Behind the numbers: Insights into large audit firm sampling policies. Accounting Horizons, 29, 61-82. https://doi.org/10.2308/acch-50921

Collopy, F., \& Armstrong, J. (1992). Rule-Based forecasting: Development and validation of an expert systems approach to combining time series extrapolations. Management Science, 38, 1394-1414. https://doi.org/10.1287/mnsc.38.10.1394

Fildes, R., Goodwin, P., \& Lawrence, M. (2006). The design features of forecasting support systems and their effectiveness. Decision Support Systems, 42, 351-361. https://doi.org/10.1016/j.dss.2005.01.003

Imoniana, J., Antunes, M. T., Mattos, S., \& Maciel, E. (2012). The analytical review procedures in audit: An exploratory study. Advances in Scientific and Applied Accounting, 5, 282-303. https://doi.org/10.14392/ASAA/2012050206

ISA (2009) International Standard on Auditing: 520 Analytic Procedures, International Auditing and Assurance Standards Board IAASB ${ }^{\circledR}$ [online], http://www.iaasb.org/

JMP ${ }^{\circledR}$ 6. (2005). Statistics and Graphics Guide. SAS Institute Inc. ISBN 1-59047-816-9

Kennedy, J., \& Mills, R. (1992). Post-Completion auditing: A source of strategic direction? Management Accounting , 70, 26-28.

Makridakis, S., Andersen, A., Carbone, R., Fildes, R., Hibon, M., Lewandowski, R., \& Winkler, R. (1982). The accuracy of extrapolation (time series) methods: Results of a forecasting competition. Journal of forecasting, 1 , 111-153. https://doi.org/10.1002/for.3980010202

O’Donnell, E., \& Perkins, J. (2011). Assessing risk with analytical procedures: Do systems-thinking tools help auditors focus on diagnostic patterns? Auditing: A Journal of Practice \& Theory, 30, 273-283. 
https://doi.org/10.2308/ajpt-10148

Public Company Accounting Oversight Board [PCAOB]. (2007). Auditing Standard No. 5: An Audit of Internal Control Over Financial Reporting That Is Integrated with An Audit of Financial Statements, https://pcaobus.org/Standards/Auditing/Pages/Auditing_Standard_5.aspx

Stanley, J., Brandon, D., \& McMillan, J. (2015). Does lowballing impair audit quality? Evidence from client accruals surrounding analyst forecasts. Journal of Accounting and Public Policy, 34, 625-645.

https://doi.org/10.1016/j.jaccpubpol.2015.05.007

Note 1: See for more details on the Analytic Procedures imperative as offered by Audit Standard 5 of the PCAOB: Sections: 9, 21, 23, $26 \& 37$ <https://pcaobus.org/Standards/Auditing/Pages/Auditing_Standard_5.aspx>

Note 2: In this regard we will use the OLS regression model that was introduced by The AICPA in Audit Guide (2012): Analytical Procedures, a deliverable of the Clarity Project. <http://www.aicpa.org/InterestAreas/FRC/AuditAttest/Pages/ImprovingClarityASBStandards.aspx>

\section{Appendix}

Table of Series Identification Numbers from Adya \& Lusk (2016) also Makridakis et al. (1982)

\begin{tabular}{rrrrrrrrrr}
\hline 14 & 17 & 24 & 27 & 28 & 34 & 37 & 38 & 44 & 47 \\
48 & 54 & 57 & 58 & 64 & 67 & 68 & 74 & 77 & 78 \\
84 & 87 & 88 & 94 & 97 & 98 & 104 & 107 & 108 & 114 \\
117 & 118 & 124 & 127 & 128 & 134 & 137 & 138 & 144 & 147 \\
148 & 154 & 157 & 158 & 164 & 167 & 168 & 174 & 177 & 178 \\
\hline
\end{tabular}

\section{Copyrights}

Copyright for this article is retained by the author(s), with first publication rights granted to the journal.

This is an open-access article distributed under the terms and conditions of the Creative Commons Attribution license which permits unrestricted use, distribution, and reproduction in any medium, provided the original work is properly cited. 